\title{
Finding the ideal biomaterial for aortic valve repair with ex vivo porcine left heart simulator and finite element modeling
}

\author{
Hadi Daood Toeg, MD, MSc, ${ }^{\mathrm{a}}$ Ovais Abessi, MEng, ${ }^{\mathrm{b}}$ Talal Al-Atassi, MD, MPH, ${ }^{\mathrm{a}}$ \\ Laurent de Kerchove, MD, ${ }^{\mathrm{c}}$ Gebrine El-Khoury, $\mathrm{MD},{ }^{\mathrm{c}}$ Michel Labrosse, $\mathrm{PhD},{ }^{\mathrm{b}}$ and \\ Munir Boodhwani, MD, MMSc ${ }^{a}$
}

\begin{abstract}
Objectives: Aortic valve (AV) repair (AVr) has become an attractive alternative to AV replacement for the correction of aortic insufficiency; however, little clinical evidence exists in determining which biomaterial at AVr would be optimal. Cusp replacement in $\mathrm{AVr}$ has been associated with increased long-term $\mathrm{AVr}$ failure. We measured the hemodynamic and biomaterial properties using an ex vivo porcine $\mathrm{AVr}$ model with clinically relevant biomaterials and generated a finite element model to ascertain which materials would be best suited for valve repair.
\end{abstract}

\begin{abstract}
Methods: Porcine aortic roots with intact AVs were placed in a left heart simulator mounted with a high-speed camera for baseline valve assessment. The noncoronary cusp was excised and replaced with autologous porcine pericardium, glutaraldehyde-fixed bovine pericardial patch (Synovis), extracelluar matrix scaffold (CorMatrix), or collagen-impregnated Dacron (Hemashield). The hemodynamic parameters were measured for a range of cardiac outputs (2.5-6.5 L/min) after repair. The biomaterial properties and St Jude Medical pericardial patch were determined using pressurization experiments. Finite element models of the AV and root complex were constructed to determine the hemodynamic characteristics and leaflet stresses.
\end{abstract}

Results: The geometric orifice areas after repair were significantly reduced in the Hemashield $(P<.05)$ and CorMatrix $(P=.0001)$ groups. Left ventricular work increased with increasing cardiac output $(P=.001)$ in unrepaired valves, as expected, and was similar among all biomaterial groups. Finite element modeling of the biomaterials displayed differences in the percentage of changes in total Von Mises stress for both replaced (noncoronary cusp) and nonreplaced left and right cusps with the St Jude Medical pericardial patch (+4\%, $+24 \%)$ and autologous porcine pericardium $(+5,+26 \%)$, with a lower percentage of changes than for the bovine pericardial patch $(+12 \%,+27 \%)$, Hemashield $(+30 \%,+9 \%)$, and CorMatrix $(+13 \%,+32 \%)$.

Conclusions: The present study has shown that postrepair left ventricular work did not increase despite a decrease in geometric orifice areas in the Hemashield and CorMatrix groups. The autologous porcine pericardium and St Jude Medical pericardial patch had the closest profile to normal AVs; therefore, either biomaterial might be best suited. Finally, the increased stresses found in the bovine pericardial patch, Hemashield, and CorMatrix groups might, after prolonged tensile exposure, be associated with late repair failure. (J Thorac Cardiovasc Surg 2014;148:1739-45)

\section{Supplemental material is available online.}

Aortic valve repair (AVr) has evolved into an attractive strategy for the correction of aortic insufficiency (AI)

\footnotetext{
From the Division of Cardiac Surgery, ${ }^{\mathrm{a}}$ University of Ottawa Heart Institute, Ottawa, Ontario, Canada; Department of Mechanical Engineering, ${ }^{\mathrm{b}}$ University of Ottawa, Ottawa, Ontario, Canada; and Department of Cardiovascular and Thoracic Surgery, ${ }^{\mathrm{c}}$ Cliniques Universitaires Saint-Luc, Brussels, Belgium.

The present study was partially supported by the Natural Sciences and Engineering Research Council of Canada for Discovery (grant 312065-2012 to M.L.).

Disclosures: Authors have nothing to disclose with regard to commercial support.

Received for publication Feb 21, 2014; revisions received April 13, 2014; accepted

for publication May 2, 2014; available ahead of print June 13, 2014.

Address for reprints: Munir Boodhwani, MD, MMSc, Division of Cardiac Surgery,

University of Ottawa Heart Institute, 40 Ruskin St, Ottawa, ON K1Y 4W7, Canada

(E-mail: mboodhwani@ottawaheart.ca).

$0022-5223 / \$ 36.00$

Copyright (c) 2014 by The American Association for Thoracic Surgery

http://dx.doi.org/10.1016/j.jtcvs.2014.05.004
}

compared with valve replacement. ${ }^{1}$ Successful repair can be achieved if the surgeon addresses the specific anatomic defects with high surgical flexibility, knowledge, and adequate surgical tools in the armamentarium. ${ }^{2}$ Attempts to replace cusp tissue with biologic material have occurred since the 1960s with materials such as dura mater, fascia lata, and bovine pericardium. ${ }^{3}$ Several groups have attempted to mimic autologous aortic valve (AV) tissue with biomechanical engineering strategies such as tissue bioreactors, ${ }^{4}$ and others have studied commercially available biomaterials in various in vitro and in vivo settings. ${ }^{5-7}$

The glutaraldehyde-treated bovine pericardial patch and treated and untreated autologous human pericardial tissue are common biomaterials used in both cardiac and vascular surgery. ${ }^{1,8,9}$ Patch repair can be used in a variety of settings. These include repair of cusp perforation due to healed endocarditis or for cusp restoration after resection of a calcified or restrictive raphe of a bicuspid AV. A patch can also be used to improve cusp coaptation in pediatric $\mathrm{AVr}$ or 


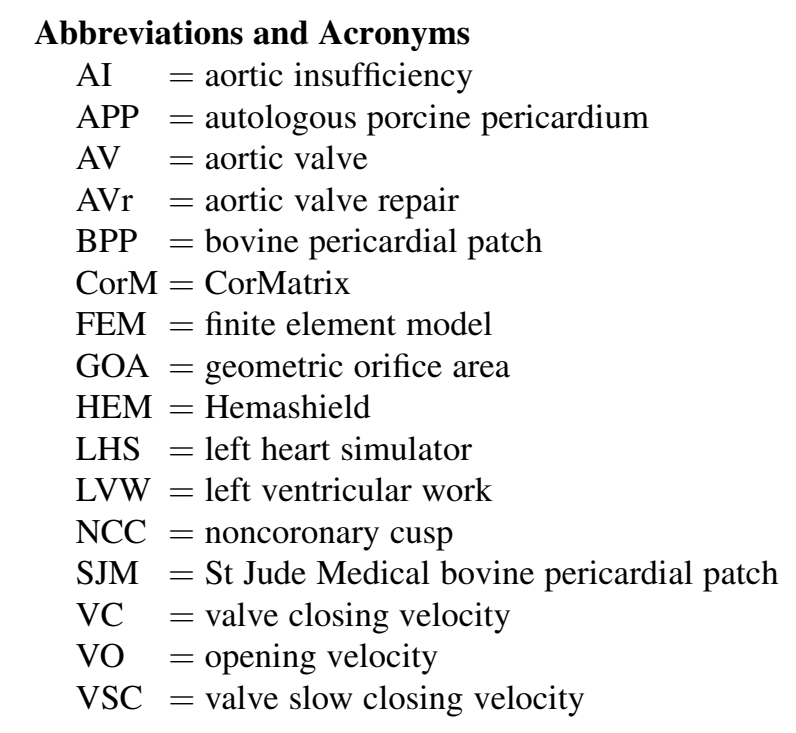

to reconstruct a commissure when creating a bicuspid valve from a unicuspid AV or a tricuspid valve from a bicuspid $\mathrm{AV} .^{8}$

An increased risk of long-term recurrent AI has been associated with the use of a patch in a number of studies ${ }^{8,10}$ that used a variety of patch materials. This might have resulted from the limitations of the existing biomaterials available for cusp reconstruction. Thus, although the perfect biomaterial has remained elusive, we sought to ascertain which materials would be best suited for cusp restoration during $\mathrm{AVr}$. Using an AV-cusp restoration ex vivo porcine left heart simulator model, we measured the hemodynamics and biomaterial properties and developed a finite element model (FEM) comparing native porcine AVs and repaired $\mathrm{AVs}$ using clinically relevant biomaterials.

\section{METHODS}

\section{Ex Vivo Porcine AV Repair Model}

Fresh aortic roots $(\mathrm{n}=25)$, with $6 \mathrm{~cm}$ of the ascending aorta, were dissected from healthy porcine (donated by a local commercial supplier), and the coronary arteries were ligated. All aortic roots were connected to the ViVitro Left Heart Simulator (LHS; ViVitro Systems, Inc, Victoria, British Columbia, Canada) for baseline valve measurements acting as paired controls. After the baseline recordings, a transverse aortotomy was created $1 \mathrm{~cm}$ above the sinotubular junction, and the noncoronary cusp (NCC) was resected, leaving a 3-mm cuff of tissue attached to the aortic wall. An outline of the excised cusp was used to create a 2-mm oversize cusp tissue from various biomaterials. The biomaterial was then connected, at both lateral edges, with two 6-0 polypropylene sutures (Ethicon Endosurgery, Johnson \& Johnson, Inc, Montreal, Ontario, Canada). The patch was sutured using a continuous running suture. The aortotomy was closed in double layers with 5-0 polypropylene (Ethicon Endosurgery).

\section{Biomaterials}

We used 4 clinically relevant materials in the present study. The first material was derived from fresh porcine pericardial tissue taken from the same porcine heart to which the valve repair was performed. The biomaterial was kept in $0.9 \%$ normal saline and has been referred to as autologous porcine pericardium (APP). Next, a collagen-impregnated double-woven Dacron Hemashield patch (HEM; Maquet, Rastatt, Germany) was used. The next material used was CorMatrix (CorM; CorMatrix, Roswell, Ga): a biomaterial derived from porcine small intestinal submucosa and used for the repair of certain cardiac defects. ${ }^{11}$ Finally, with $>30$ years of cardiovascular implantation experience, the PeriGuard bovine pericardial patch (BPP; Synovis Surgical Innovations, Deerfield, Ill) was used. All these biomaterials were used in the LHS model. In addition to the listed biomaterials, we considered another bovine pericardial patch donated by St Jude Medical (SJM; St Jude Medical, Inc, St Paul, Minn) to perform our FEM experiments (see the sections "Finite Element Model" and "Simulation of Leaflet Repair"). The SJM Pericardial Patch with EnCap AC Technology (St Jude Medical) is a thinner, flexible bovine pericardial patch with a different anticalcification treatment than that used for $\mathrm{BPP}^{15}$ (Table E1).

\section{LHS Measurements}

The Vivitro LHS (ViVitro Systems, Inc) was used as a pulse duplicator with the capabilities of measuring flow and pressure and generating a wide range of cardiac outputs. This LHS consists of a piston pump system, a viscoelastic impedance adapter for afterload generation, an adjustable left ventricular (LV) membrane, flow and pressure monitoring systems, a waveform generator, and a data acquisition system. In addition, an adjustable jig was used to attach the repaired aortic roots to the system. A highspeed camera (Phantom, version 4.2, Vision Research, Inc, Wayne, NJ) was connected to an endoscope and light source to record images of valvular leaflet motion from the aortic side. Additional details have been described previously by our group. $^{12}$

For the valve hemodynamic measurements, the unrepaired or repaired aortic root was connected at room temperature to the LHS. The system, containing $0.9 \%$ normal saline (density, $0.9 \mathrm{~g} / \mathrm{mL}$; and viscosity, $1 \mathrm{mPa} / \mathrm{s}$ ) as the fluid medium, was pressurized from $0 \mathrm{~mm} \mathrm{Hg}$ to a systolic pressure of $120 \mathrm{~mm} \mathrm{Hg}$ and diastolic pressure of $80 \mathrm{~mm} \mathrm{Hg}$. The heart rate was set at 70 beats/min for all experiments, and the cardiac output was varied from 2.5 to $6.5 \mathrm{~L} / \mathrm{min}$. The parameters measured included the valve opening velocity (VO), valve slow closing velocity (VSC), valve closing velocity (VC), maximum geometric orifice area (GOA), and LV work (LVW). The AI fraction was insignificant in all treated aortic roots.

\section{Finite Element Model}

Using the procedure outlined in our previous study, ${ }^{12}$ a FEM of a typical unpressurized porcine AV with the root was built with the dimensions listed in Tables E1 and E2. The structured hexahedral FEM mesh consisted of approximately 11,800 nodes and 8600 elements. The valve model was studied for 1 cardiac cycle by application of set pressure pulses. $^{12}$ Because the analysis started from the unpressurized geometry, the pressure was ramped from 0 to $\leq 80 \mathrm{~mm} \mathrm{Hg}$ before the physiologic cardiac cycle started in early systole. Although the normal duration of diastole is approximately 0.60 second, it was shortened to 0.10 second in the simulation to save computational time. In addition, the simulation time was $1 / 10$ of the real time, because analyses with real or scaled time yielded results within $2 \%$ of each other owing to the comparatively small inertial loads. All numeric analyses were performed using commercial FEM software LS-Dyna, version 971 (Livermore Software Technology Corp, Livermore, Calif). The mass density of the aortic and leaflet tissues was set at $1000 \mathrm{~kg} / \mathrm{m}^{3}$. The time step was automatically set and updated by LS-Dyna to achieve numeric stability of the solution. The longitudinal stretch ratio was set to the normal physiologic value of $1.20 .^{13}$ The tissues of the aorta and aortic leaflets were both modeled as hyperelastic, transversely isotropic materials using a Fung-like model with strain energy function: 


$$
\begin{aligned}
W= & \frac{C_{1}}{2}\left[\operatorname { e x p } \left(c_{2} E_{\theta}^{2}+c_{3}\left(E_{z}^{2}+E_{r}^{2}+E_{r z}^{2}+E_{z r}^{2}\right)\right.\right. \\
& \left.\left.+c_{4}\left(E_{\theta z}^{2}+E_{\theta r}^{2}+E_{r \theta}^{2}+E_{z \theta}^{2}\right)\right)-1\right]+\frac{P}{2}(J-1),
\end{aligned}
$$

where $c_{1}, \ldots, c_{4}$ are material constants, $E \ldots$ are deformations (Green strain components modified to only include the effects of volumetric work), and the subscripts $\theta, z$, and $r$ refer to the circumferential, longitudinal, and radial directions, respectively. $P$ is a Lagrange multiplier numerically enforcing the material near-incompressibility such that $J$, the determinant of the deformation gradient tensor, is almost equal to 1 . Although this material model was initially developed to represent myocardial tissue, it has been shown to give accurate representations of human aortic and leaflet tissue. ${ }^{14}$ The material constants for the original porcine model are listed in Table E1. The unknown constant $c_{4}$ in the material model was assumed to equal $c_{3}$ (see Labrosse and colleagues ${ }^{14}$ for our justification).

\section{Simulation of Leaflet Repair}

With all other parameters unchanged, the thickness and material properties of the NCC in the original porcine valve model were modified to represent those of the biomaterials considered in the present study. The biomaterial properties were measured using static pressurization experiments. Each sample was sutured into a cylinder, cannulated at 1 end, capped at the other, and placed in normal saline at room temperature. The sample was held horizontally by the cannulated end, which was connected to a saline solution head, whose pressure was measured using a digital manometer (33500-082, VWR International, Mississauga, ON, Canada). The sample was preconditioned by three 1-minute pressurizations from $0 \mathrm{~mm} \mathrm{Hg}$ to $160 \mathrm{~mm} \mathrm{Hg}$ to $0 \mathrm{~mm} \mathrm{Hg}$. The elongation of the sample was monitored using a digital camera (Powershot IS2, Canon, Tokyo, Japan), with 2 markers affixed to it. For the measurement run, the pressure was slowly stepped up from 0 to $160 \mathrm{~mm} \mathrm{Hg}$ in $20-\mathrm{mm} \mathrm{Hg}$ increments. Cross-sectional views of the loaded sample were acquired using an ultrasound external probe (Terason SMARTProbe, $5-8 \mathrm{MHz}, 4-\mathrm{cm}$ linear array "T" probe; Teratech Corp, Burlington, Mass) driven by the personal computer-based TelaVet 1000 system (Classic Medical, Tequesta, Fla). The corresponding images synchronized with the pressure steps were stored. Processing of similar data has been previously detailed ${ }^{14}$; in the present study, it yielded the properties listed in Table E1. Figure 1 illustrates the circumferential and radial direction stress-strain relationships for the various biomaterials (color coded) under equibiaxial planar testing driven in tension. In all the FEMs, the maximum values of mechanical Von Mises stress in the leaflets were determined, with their location and timing. Von Mises stress summarizes in 1 number the stresses present in different directions of the material at 1 specific location. In addition, to evaluate the VO and VS, the GOA of the valve was measured as a function of time from the calculated leaflet motions throughout 1 cardiac cycle. This was done using the postprocessor of LS-Dyna to take top view snapshots of the valve at regular intervals during the cardiac cycle and measuring the projected area left open by the leaflets.

\section{Statistical Analysis}

Statistical analysis was performed using STATA, version 12, statistical program (StataCorp LP, College Station, Tex). Nonparametric data are expressed as the median and interquartile range. Wilcoxon-signed rank tests were used to determine the significance between the paired sample sets, and the Kruskal-Wallis rank test was used for $>2$ sample sets.

\section{RESULTS}

A total of 25 fresh porcine aortic roots underwent baseline measurements. The AVs subsequently underwent randomized allocation to NCC excision and cusp replacement with APP $(n=7)$, HEM $(n=7)$, CorM $(n=5)$, and
BPP $(n=6)$. Each unrepaired and repaired AV underwent 3 sample runs at set cardiac outputs of 2.5, 5.0, and $6.5 \mathrm{~L} / \mathrm{min}$.

\section{Hemodynamic Results From LHS}

For appropriate comparisons, each treatment sample was compared with its original valve measurements (paired experiments). Compared with the baseline valve hemodynamics, the APP biomaterial demonstrated significantly lower VO $(P=.02)$ and VC $(P=.03)$ values (Table 1$)$. Although not statistically significant, the postrepair GOA was reduced from $5.74 \mathrm{~cm}^{2}$ (range, 5.29-6.87) to 5.25 $\mathrm{cm}^{2}$ (range, 4.16-5.99; $P=.08$ ). Despite this decrease in the GOA, the LVW after repair did not change $(P=.6)$. After replacing NCCs with HEM biomaterial, a significant reduction was seen in the GOA (from $5.91 \mathrm{~cm}^{2}$ [range, 5.58-6.43] to $5.06 \mathrm{~cm}^{2}$ [range, 4.24-5.73]; $P=.05$ ) and $\mathrm{VC}$ (from $63 \mathrm{~cm} / \mathrm{s}$ [range, 59-71] to $48 \mathrm{~cm} / \mathrm{s}$ [range, 35 61]; $P=.01)$ compared with the baseline values (Table 1). All other parameters, including VO, VSC, and LVW, did not significantly change $(P>.3)$. The postrepair valve hemodynamics with CorM demonstrated significant reductions in the VO $(P=.0001)$, VSC $(P=.01)$, VC $(P=.0001)$, and GOA $(P=.0001$; Table 1$)$. Despite these findings, the LVW was similar between the baseline and repaired valves (from $1033 \mathrm{~mJ}$ [range, 694-1221] to $1086 \mathrm{~mJ}$ [range, 727-1241]; $P=.4$ ). Finally, after comparing the BPP-repaired valves with baseline valve hemodynamics, no significant differences were observed in VO $(P=.4)$, VSC $(P=.08)$, VC $(P=.8)$, GOA $(P=.5)$, or LVW $(P=.4$; Table 1$)$.

As expected, when the native nonrepaired porcine valve hemodynamics at cardiac output settings of 2.5, 5.0, and $6.5 \mathrm{~L} / \mathrm{min}$ were compared, the LVW and VO increased with increasing cardiac output $(P=.001$ and $P=.002$, respectively; Table 2). The differences for all other parameters, including GOA, were not statistically significant $(P>.05$; Table 2$)$.

\section{Finite Element Model}

All FEMs opened and closed properly. The hemodynamics (VO, VC) of the repaired valves were not distinguishable from those of the original valves (Table 3). The repaired valves opened and closed more slowly than did the original valve by, at most, $9 \%$. The GOA of the repaired valves was $6 \%$ to $9 \%$ smaller than that of the original unrepaired valve. In the repaired valves, all biomaterials produced different maximum Von Mises stresses in all leaflets (NCC and left and right nonreplaced coronary cusps; Table 3). The replaced leaflets (NCC) with the smallest stresses were made of SJM and APP. In the NCC, the stresses in SJM and APP were $4 \%$ and $5 \%$ greater than the stresses in the native leaflets, respectively. In contrast, the replaced leaflets that experienced the greatest stresses 


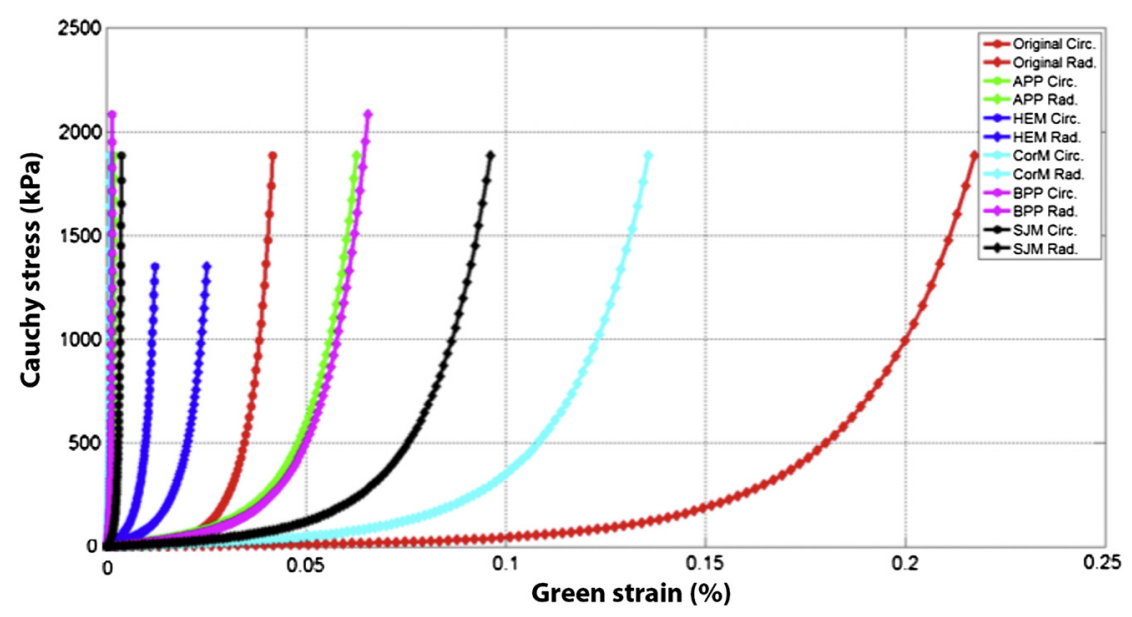

FIGURE 1. Representative circumferential and radial stress-strain properties of the original leaflets and corresponding biomaterials derived from equibiaxial planar testing (Cauchy stress, kPa; Green strain, \%). Original, Native unrepaired valve; Rad, radial direction; Circ, circumferential direction; $A P P$, autologous porcine pericardium; HEM, Hemashield; CorM, CorMatrix; BPP, bovine pericardial patch; SJM, St Jude Medical patch.

consisted of HEM $(+30 \%)$, CorM $(+13 \%)$, and BPP $(+12 \%)$. Owing to the interaction between the AV cusps during coaptation, the stresses in the nonreplaced leaflets were also affected by the material used. The smallest increases in stress experienced by the native leaflets (left and right nonreplaced cusps) were $9 \%$ when HEM was used for the replaced leaflet, $24 \%$ with SJM, and $26 \%$ with APP. The greatest increases in stress were 32\% with CorM and $27 \%$ with BPP. Finally, after combining the Von Mises stresses of both replaced (NCC) and nonreplaced (left and right) cusps, the SJM biomaterial had the lowest increase in stress at $+28 \%$ (3968 $\mathrm{kPa})$, followed by APP with $+31 \%(4047 \mathrm{kPa})$ compared with the native unrepaired model. The greatest increase in combined Von Mises stress was found in the CorM group $(+45 \% ; 4282 \mathrm{kPa})$ followed by the HEM $(+39 \% ; 4158 \mathrm{kPa})$ and BPP $(+39 \%$; $4158 \mathrm{kPa}$ ) groups. An illustration of the Von Mises stresses experienced on the AV cusps is depicted in Figure 2.

\section{DISCUSSION}

Given the limited durability of $\mathrm{AVr}$ in the setting of patch replacement for cusp tissue, our objective was to determine which biomaterials would be best suited for AV cusp repair. Using an ex vivo porcine AV LHS model to assess the valvular hemodynamics and FEM to assess leaflet stress, we compared a variety of clinically relevant biomaterials. We found that the postrepair LVW did not increase, despite a decrease in GOAs in the HEM and CorM groups. Our ex vivo LHS model suggested that BPP would be best suited for AVr. However, the FEM demonstrated increased cusp stresses in the BPP, HEM, and CorM groups. The increased cusp stresses, after a significant amount of exposed force, might be associated with late repair failure. APP and the thinner SJM pericardial patch had the closest profile to normal AVs and would be best suited for AV cusp repair.

Although clinical experience has suggested increase rates of recurrent $\mathrm{AI}$ in repaired $\mathrm{AVs}$ when patch material has

TABLE 1. Comparison of unrepaired (baseline controls) and repaired valve hemodynamics for biomaterials tested with combined cardiac outputs of $2.5,5.0$, and $6.5 \mathrm{~L} / \mathrm{min}$

\begin{tabular}{|c|c|c|c|c|c|}
\hline Material & VO $(\mathrm{cm} / \mathrm{s})$ & $\mathrm{VSC}(\mathrm{cm} / \mathrm{s})$ & $\mathrm{VC}(\mathrm{cm} / \mathrm{s})$ & Maximum GOA $\left(\mathrm{cm}^{2}\right)$ & LVW (mJ) \\
\hline Control & $157(140-213)$ & $6.3(4.5-8)$ & $68(53-76)$ & $5.74(5.29-6.87)$ & $1002(703-1238)$ \\
\hline APP & $113(67-163)$ & $5.35(2.6-6.9)$ & $48(44-55)$ & $5.25(4.16-5.99)$ & $1016(932-1229)$ \\
\hline$P$ value & .03 & .2 & .02 & .08 & .6 \\
\hline Control & $136(109-167)$ & $3.3(0.3-7.5)$ & $63(59-71)$ & $5.91(5.58-6.43)$ & $1018(689-1234)$ \\
\hline HEM & $120(100-164)$ & $4.4(2.4-6.7)$ & $48(35-61)$ & $5.06(4.24-5.73)$ & $1106(763-1249)$ \\
\hline$P$ value & .3 & .4 & .01 & .05 & .3 \\
\hline Control & $219(188-241)$ & $6.2(5.2-13.1)$ & $81(69-86)$ & $6.08(5.82-6.83)$ & $1033(694-1221)$ \\
\hline CorM & $111(76-150)$ & $4.3(1.5-6)$ & $52(44-63)$ & $5.27(4.57-5.56)$ & $1086(727-1241)$ \\
\hline$P$ value & .0001 & .01 & .0001 & .0001 & .4 \\
\hline Control & $177(153-213)$ & $7.25(4.7-11.3)$ & $65.5(58-83)$ & $5.45(4.99-6.36)$ & $1039(709-1222)$ \\
\hline BPP & $173.5(127-195)$ & $4.05(3.3-7.6)$ & $72.5(57-80)$ & $5.08(4.54-6.59)$ & $1059(730-1253)$ \\
\hline$P$ value & .4 & .08 & .8 & .5 & .4 \\
\hline
\end{tabular}

$V O$, Valve opening velocity; $V S C$, valve slow closing velocity; $V C$, valve closing velocity; $G O A$, geometric orifice area; $L V W$, left ventricular work; $A P P$, autologous porcine pericardium ( $\mathrm{n}=21) ; H E M$, Hemashield $(\mathrm{n}=21)$; CorM, CorMatrix $(\mathrm{n}=15) ; B P P$, bovine pericardial patch $(\mathrm{n}=18)$. 
TABLE 2. Valve hemodynamics in unrepaired porcine valves at different cardiac output

\begin{tabular}{|c|c|c|c|c|c|}
\hline \multirow[b]{2}{*}{ Measure } & \multirow[b]{2}{*}{ Combined $(n=75)$} & \multicolumn{3}{|c|}{$\mathrm{CO}(\mathrm{L} / \mathrm{min})$} & \multirow[b]{2}{*}{$P$ value } \\
\hline & & $2.5(n=25)$ & $5.0(n=25)$ & $6.5(n=25)$ & \\
\hline $\mathrm{VO}(\mathrm{cm} / \mathrm{s})$ & $171(140-213)$ & $145(116-159)$ & $181(142-219)$ & $194(164-220)$ & .002 \\
\hline $\operatorname{VSC}(\mathrm{cm} / \mathrm{s})$ & $5.9(2.9-8.8)$ & $7.5(5.5-9)$ & $5.8(1.3-9.5)$ & $5.2(2.2-7.2)$ & .12 \\
\hline $\mathrm{VC}(\mathrm{cm} / \mathrm{s})$ & $68(59-79)$ & $61(48-73)$ & $68(60-83)$ & $76(63-83)$ & .05 \\
\hline Maximum GOA $\left(\mathrm{cm}^{2}\right)$ & $5.82(5.37-6.5)$ & $5.8(5.4-5.99)$ & $6.06(5.32-6.56)$ & $5.83(5.31-6.67)$ & .5 \\
\hline LVW (mJ) & $1029(703-1222)$ & $686(661-703)$ & $1032(1000-1051)$ & $1249(1229-1283)$ & .001 \\
\hline
\end{tabular}

$C O$, Cardiac output; $V O$, valve opening velocity; $V S C$, valve slow closing velocity; $V C$, valve closing velocity; $G O A$, geometric orifice area; $L V W$, left ventricular work.

been used for reconstruction, ${ }^{8,16}$ translating this finding to a clinically relevant preclinical model poses significant challenges. Given the nature of AV cusp repair, a large animal model would be required, because small animal models would be associated with significant technical limitations. The absence of large animal models for AV disease, the challenges in performing complex $\mathrm{AV}$ interventions in such animals, and the need to allow these animals to survive for months (if not longer) to observe clinically relevant changes are some of the challenges. As such, models that provide detailed information on valve dynamics, complemented with data on valve stresses, will be the best surrogate indicators available to ascertain the long-term performance of cusp repair materials. Moreover, similar approaches have been used in the development of prosthetic valves. ${ }^{17} \mathrm{We}$, therefore, used such a combination in the present study.

The clinical applications of autologous human pericardial tissue have included $\mathrm{AVr}$, aortic root enlargement, and LV aneurysm repair. ${ }^{1,9,10,18}$ Because APP has characteristics similar to those of human pericardial tissue, APP was used in the present study as a surrogate to human pericardium. ${ }^{19}$ The APP used was derived from fresh porcine pericardium and had poor handling qualities owing to its fragile structure. The postrepair VO $(P=.03)$ and $\mathrm{VC}(P=.02)$ were significantly reduced compared with those of the unrepaired valves; however, the GOA and LVW were unchanged. Thus, APP would be a reasonable biomaterial choice because it demonstrates similar native valvular hemodynamics. Next, when the HEM patch was used to replace the NCC, the VC $(P=.01)$ and GOA $(P<.05)$ were significantly reduced, but the other parameters, including LVW, VSC, and VO, were similar. This suggests that, despite the lack of increased LVW, the restricted noncompliant nature of the HEM material led to slower VC and a smaller GOA. Although the HEM has been used for aortic root enlargement and LV aneurysm repair and has also been known for its mechanical strength ${ }^{20}$ and long-term durability, ${ }^{21}$ it has not been used for AVr in the clinical setting. ${ }^{22}$ Our results have shown that it is likely that it should continue not being used for AVr.

With the use of CorM for $\mathrm{AVr}$, all valvular hemodynamic parameters, including VO $(P=.0001)$, VSC $(P=.01), \mathrm{VC}$ $(P=.0001)$, and GOA $(P=.0001)$ were significantly reduced compared with the baseline values. A slight increase was found in LVW after CorM repair, but the difference did not reach statistical significance $(P=.4)$. From all these early hemodynamic measurements, the CorM biomaterial might not be the most ideal product for AVr. CorMatrix is an approved sheet for the use in cardiac surgical septal repair ${ }^{23}$ and pericardial closure ${ }^{24}$; however, little information has been provided in published studies reporting on clinical applications for AVr. ${ }^{11}$ In addition, our ex vivo and FEMs evaluated CorMatrix before autologous cell seeding, which might alter its biomaterial properties and might not represent the long-term repair characteristics. After repair with BPP, the valvular hemodynamics did not change across all parameters (VO, VSC, VC, GOA, and LVW). With the easy handling properties, these findings suggest that BPP is a reasonable choice for biomaterial selection in AVr. Some studies have demonstrated good short-term outcomes after cusp restoration with the bovine pericardial patch ${ }^{25}$; however, long-term recurrent $\mathrm{AI}$ was

TABLE 3. Summary of FEM simulation results

\begin{tabular}{|c|c|c|c|c|c|c|}
\hline Measure & Original & APP & HEM & CorM & BPP & SJM \\
\hline $\mathrm{VO}(\mathrm{cm} / \mathrm{s})$ & 70 & $65(-7)$ & $64(-9)$ & $66(-6)$ & $65(-7)$ & $66(-6)$ \\
\hline $\mathrm{VC}(\mathrm{cm} / \mathrm{s})$ & 55 & $53(-4)$ & $52(-5)$ & $53(-4)$ & $55(0)$ & $53(-4)$ \\
\hline Maximum GOA $\left(\mathrm{cm}^{2}\right)$ & 5.6 & $5.2(-7)$ & $5.1(-9)$ & $5.3(-6)$ & $5.2(-7)$ & $5.3(-6)$ \\
\hline $\mathrm{NCC}(\mathrm{kPa})$ & 1746 & $1841(+5)$ & $2263(+30)$ & $1973(+13)$ & $1946(+12)$ & $1809(+4)$ \\
\hline $\mathrm{L} / \mathrm{R}$ cusps $(\mathrm{kPa})$ & 1746 & $2206(+26)$ & $1895(+9)$ & $2309(+32)$ & $2212(+27)$ & $2159(+24)$ \\
\hline Combined stress $(\mathrm{kPa})$ & 3492 & $4047(+31)$ & $4158(+39)$ & $4282(+45)$ & $4158(+39)$ & $3968(+28)$ \\
\hline
\end{tabular}

Data in parentheses are percentages. APP, Autologous porcine pericardium; HEM, Hemashield; CorM, CorMatrix; BPP, bovine pericardial patch; SJM, St Jude Medical bovine pericardial patch; $V O$, valve opening velocity; $V C$, valve closing velocity; $G O A$, geometric orifice area; $N C C$, maximum Von Mises stress in replaced noncoronary cusp; $L / R$ cusps, maximum Von Mises stress in left and right nonreplaced coronary cusps. 

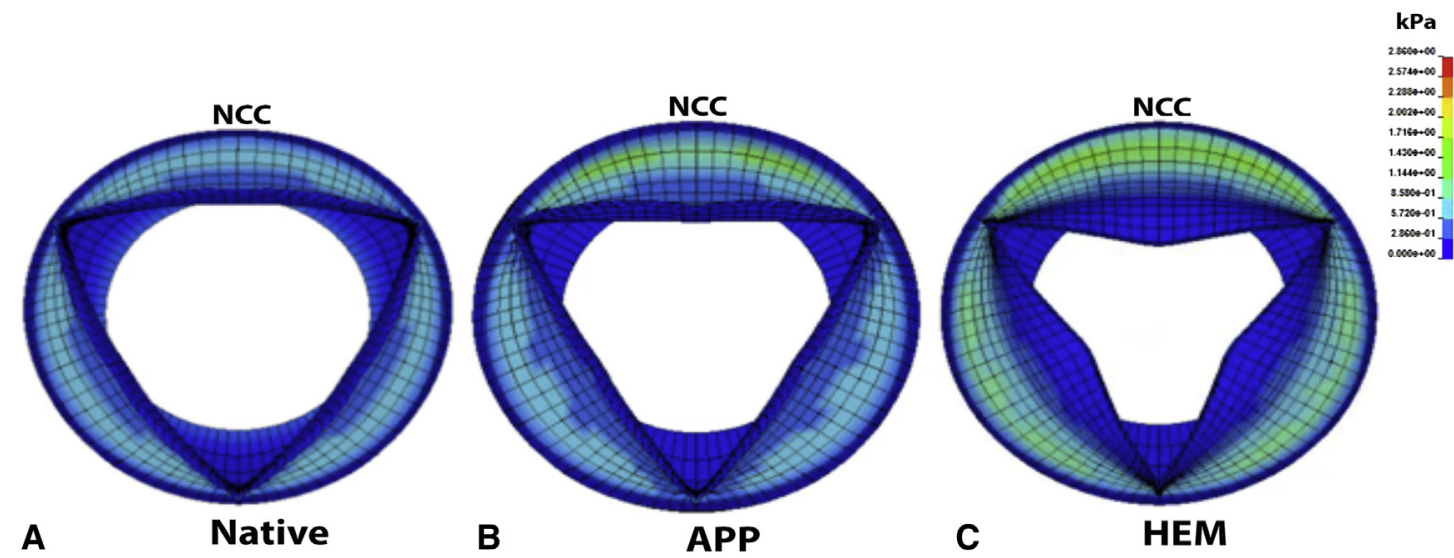

FIGURE 2. Illustration of the Von Mises stresses on the replaced cusps (noncoronary cusp [NCC]) with finite element modeling demonstrating (A) native unrepaired valve; $\mathrm{B}$, autologous porcine pericardium (APP), and (C) Hemashield (HEM). Blue areas represent minimal to no stress (0 kPa); light blue, green, and yellow represent increasing levels of stress (maximum $7.774 \mathrm{kPa}$ ). Increased maximal stress was seen at the suture lines in the replaced NCCs for (B) APP, and (C) HEM compared with the (A) native unrepaired valve.

demonstrated in a predominately porcine pericardial patch technique used for cusp restoration. ${ }^{8,16}$ Also, fresh and dye-mediated photo-oxidation process (PhotoFix) autologous pericardium showed a trend toward better durability than glutaraldehyde-fixed bovine pericardium. However, the predictors of reoperation did not include the biomaterial used in their study (a pediatric population). ${ }^{26}$ In contrast, 1 group demonstrated that the use of a pericardial patch at $\mathrm{AVr}$ was a statistically significant predictor of reoperation, further complicating the choice of material to use. ${ }^{27}$

The valvular hemodynamic measurements from our ex vivo LHS model suggested that APP and BPP would be the best suited biomaterial candidates for $\mathrm{AVr}$; however, no information was available regarding the stress and strain experienced by the AV cusps. Our FEM demonstrated that although the $\mathrm{VO}, \mathrm{VC}$, and GOA values did not differ by $>9 \%$ (range, $0 \%-9 \%$ ) among all biomaterials and unrepaired valves, the Von Mises stresses varied significantly among the biomaterials in both the NCC (replaced cusp) and the left and right nonreplaced cusps. Von Mises stress represents a combination of all stresses acting on a particular site. ${ }^{28}$ This variable has been measured in previous studies in determining specific locations (ie, the aortic commissures) with greatest dynamic and static stresses. ${ }^{12,14,29}$ By understanding the values and patterns of valvular stresses, surgeons will be able to use better, more stresscompatible biomaterials in the reparative process, thereby reducing the incidence of AVr-related failure. ${ }^{8,10,16,30}$ The SJM bovine pericardial patch resulted in the least increase in the NCC $(+4 \% ; 1809 \mathrm{kPa})$ and left and right nonreplaced cusp $(+24 \% ; 2159 \mathrm{kPa})$ stresses. Similarly, the APP demonstrated the next least increase in the NCC $(5 \% ; 1841 \mathrm{kPa})$ and left and right nonreplaced cusp $(+26 \% ; 2206 \mathrm{kPa})$ stresses. Other groups, including the HEM, CorM, and BPP, demonstrated combined increased stresses ranging from $39 \%$ to $45 \%$ compared with baseline. Thus, from our FEM results, the SJM and APP represent the most ideal biomaterials for AVr. Although the ex vivo data suggested that BPP would be best suited for $\mathrm{AVr}$, the FEM showed that the total cusp stresses were the second greatest in this group and that it might not be the ideal biomaterial.

In the present study, we used a model that studies the detailed early hemodynamic parameters after AVr. The effect of remodeling of the biomaterial over time on cusp function was not evaluated. Future long-term experiments are warranted to establish a direct correlation between the early hemodynamic profiles of various biomaterials and their failure rate. Furthermore, other factors, including the long-term biocompatibility of the leaflet materials and inherent predisposition to calcification or degradation, were not measured, limiting our final conclusions. Other limitations included the lack of an in vivo model to evaluate the postrepair hemodynamics and the use of a mathematical FEM to derive the stress patterns. Although an in vivo model could provide more realistic settings, it would be difficult to perform the precise measurements of valve function used in the present study. Furthermore, the FEM in the present study has been used extensively in cardiac surgical studies, providing information not readily available from in vivo studies. ${ }^{14,29}$

Altogether, the results from the present study have demonstrated that postrepair LVW did not increase, despite a decrease in GOAs in the HEM and CorM groups. Although the ex vivo LHS model suggested that the BPP would be best suited for $\mathrm{AVr}$, the FEM demonstrated increased stresses in the BPP, HEM, and CorM groups, which might, after prolonged exposure to additional forces, be associated with late repair failure. Finally, APP and SJM had the closest profile to the normal AVs in both models of 
early valvular hemodynamics; therefore, the use of either biomaterial might be best suited for $\mathrm{AVr}$.

We would like to thank CorMatrix and St Jude Medical for donating their respective materials. Dr Labrosse. gratefully acknowledges the Natural Sciences and Engineering Research Council of Canada for Discovery (grant 312065-2012).

\section{References}

1. Fattouch K, Murana G, Castrovinci S, Nasso G, Mossuto C, Corrado E, et al. Outcomes of aortic valve repair according to valve morphology and surgical techniques. Interact Cardiovasc Thorac Surg. 2012;15:644-50.

2. Boodhwani M, de Kerchove L, Glineur D, Poncelet A, Rubay J, Astarci P, et al. Repair-oriented classification of aortic insufficiency: impact on surgical techniques and clinical outcomes. J Thorac Cardiovasc Surg. 2009;137:286-94.

3. Al-Halees Z, Gometza B, Duran CM. Aortic valve repair with bovine pericardium. Ann Thorac Surg. 1998;65:601-2.

4. Yang C, Sodian R, Fu P, Luders C, Lemke T, Du J, et al. In vitro fabrication of a tissue engineered human cardiovascular patch for future use in cardiovascular surgery. Ann Thorac Surg. 2006;81:57-63.

5. de Kerchove L, Vismara R, Mangini A, Fiore GB, Price J, Noirhomme P, et al. In vitro comparison of three techniques for ventriculo-aortic junction annuloplasty. Eur J Cardiothorac Surg. 2012;41:1117-23; discussion 1123-4.

6. Scharfschwerdt M, Pawlik M, Sievers HH, Charitos E. In vitro investigation of aortic valve annuloplasty using prosthetic ring devices. Eur $J$ Cardiothorac Surg. 2011;40:1127-30.

7. Rankin JS, Conger JL, Tuzun E, Winkler JA, Harms KM, Beavan LA, et al. In vivo testing of an intra-annular aortic valve annuloplasty ring in a chronic calf model. Eur J Cardiothorac Surg. 2012;42:149-54.

8. Boodhwani M, de Kerchove L, Glineur D, Rubay J, Vanoverschelde JL, Noirhomme P, et al. Repair of regurgitant bicuspid aortic valves: a systematic approach. J Thorac Cardiovasc Surg. 2010;140:276-84.e1.

9. Doss M, Sirat S, Risteski P, Martens S, Moritz A. Pericardial patch augmentation for repair of incompetent bicuspid aortic valves at midterm. Eur J Cardiothorac Surg. 2008;33:881-4.

10. Lausberg HF, Aicher D, Langer F, Schafers HJ. Aortic valve repair with autologous pericardial patch. Eur J Cardiothorac Surg. 2006;30:244-9.

11. Quarti A, Nardone S, Colaneri M, Santoro G, Pozzi M. Preliminary experience in the use of an extracellular matrix to repair congenital heart disease. Interact Cardiovasc Thorac Surg. 2012;13:569-72.

12. Labrosse MR, Lobo K, Beller CJ. Structural analysis of the natural aortic valve in dynamics: from unpressurized to physiologically loaded. J Biomech. 2010;43: 1916-22.

13. Han HC, Fung YC. Longitudinal strain of canine and porcine aortas. J Biomech. 1995;28:637-41.
14. Labrosse MR, Boodhwani M, Sohmer B, Beller CJ. Modeling leaflet correction techniques in aortic valve repair: a finite element study. J Biomech. 2011;44: 2292-8.

15. Hopkins R, Bert A, Buchholz B, Guarino K, Meyers M. Surgical patch closure of atrial septal defects. Ann Thorac Surg. 2004;77:2144-9.

16. Van Dyck M, Glineur D, de Kerchove L, El Khoury G. Complications after aortic valve repair and valve-sparing procedures. Ann Cardiothorac Surg. 2013;2: $130-9$.

17. Chaikof EL. The development of prosthetic heart valves-lessons in form and function. N Engl J Med. 2007;357:1368-71.

18. Cohen O, De La Zerda DJ, Odim J, Dinov I, Laks H. Aortic valve-sparing repair with autologous pericardial leaflet extension has low long-term mortality and reoperation rates in children and adults. Heart Surg Forum. 2007;10:E288-91.

19. Baumer TG, Powell BJ, Fenton TW, Haut RC. Age dependent mechanical properties of the infant porcine parietal bone and a correlation to the human. J Biomech Eng. 2009;131:111006.

20. Kapadia MR, Popowich DA, Kibbe MR. Modified prosthetic vascular conduits Circulation. 2008;117:1873-82.

21. Nagano N, Cartier R, Zigras T, Mongrain R, Leask RL. Mechanical properties and microscopic findings of a Dacron graft explanted 27 years after coarctation repair. J Thorac Cardiovasc Surg. 2007;134:1577-8.

22. Girardi LN, Talwalkar NG, Coselli JS. Aortic root replacement: results using the St. Jude Medical/Hemashield composite graft. Ann Thorac Surg. 1997;64: 1032-5.

23. Yanagawa B, Rao V, Yau TM, Cusimano RJ. Initial experience with intraventricular repair using CorMatrix extracellular matrix. Innovations (Phila). 2013;8: 348-52.

24. Stelly M, Stelly TC. Histology of CorMatrix bioscaffold 5 years after pericardial closure. Ann Thorac Surg. 2013;96:e127-9.

25. Tao L, Zeng XJ, Lim YP. Single cusp replacement for aortic regurgitation. Ann Thorac Surg. 2008;85:946-8.

26. Myers PO, Tissot C, Christenson JT, Cikirikcioglu M, Aggoun Y, Kalangos A Aortic valve repair by cusp extension for rheumatic aortic insufficiency in children: long-term results and impact of extension material. J Thorac Cardiovasc Surg. 2010;140:836-44.

27. Aicher D, Kunihara T, Abou Issa O, Brittner B, Graber S, Schafers HJ. Valve configuration determines long-term results after repair of the bicuspid aortic valve. Circulation. 2011;123:178-85.

28. Gouget CL, Girard MJ, Ethier CR. A constrained von Mises distribution to describe fiber organization in thin soft tissues. Biomech Model Mechanobiol. 2012;11:475-82.

29. Labrosse MR, Gerson ER, Veinot JP, Beller CJ. Mechanical characterization of human aortas from pressurization testing and a paradigm shift for circumferential residual stress. J Mech Behav Biomed Mater. 2013;17:44-55.

30. Sun L, Chandra S, Sucosky P. Ex vivo evidence for the contribution of hemodynamic shear stress abnormalities to the early pathogenesis of calcific bicuspid aortic valve disease. PLOS ONE. 2012;7:e48843. 
TABLE E1. Material properties of valve constituents and biomaterials

\begin{tabular}{lcccc}
\hline \multicolumn{1}{c}{ Material } & $\boldsymbol{c}_{\mathbf{1}}(\mathbf{k P a})$ & $\boldsymbol{c}_{\mathbf{2}}(\mathbf{k P a})$ & $\boldsymbol{c}_{\mathbf{3}}(\mathbf{k P a})$ & $\begin{array}{c}\text { Thickness } \\
(\mathbf{m m})\end{array}$ \\
\hline Porcine aorta & 6.35 & 2.62 & 3.72 & 1.9 \\
Porcine leaflet & 2.01 & 221 & 48.2 & 0.53 \\
APP & 3.00 & 11,200 & 375 & $0.53(0.12)^{*}$ \\
HEM & 2.26 & 2130 & 1060 & 0.74 \\
CorM & 3.00 & 15,700 & 111 & $0.53(0.19)^{*}$ \\
BPP & 2.59 & 15,300 & 376 & 0.48 \\
SJM & 3.00 & 4180 & 186 & $0.53(0.21)^{*}$ \\
\hline
\end{tabular}

$\overline{c_{1}, c_{2}, c_{3} \text {, Material constants (see text for details); } A P P \text {, autologous porcine pericar- }}$ dium; HEM, Hemashield; CorM, CorMatrix; BPP, bovine pericardial patch; SJM, St Jude Medical patch. *The measured values in parentheses were corrected to $0.53 \mathrm{~mm}$ for stability of the finite element simulation; the material constants were obtained using the corrected thicknesses.
TABLE E2. Unpressurized porcine aortic valve dimensions

\begin{tabular}{lc}
\hline \multicolumn{1}{c}{ Parameter } & Dimension $(\mathbf{m m})$ \\
\hline Left ventricular outflow tract & 24 \\
Sinotubular junction & 22 \\
Valve height & 17.8 \\
Maximum radius of aortic sinuses & 15 \\
Leaflet height & 17 \\
Leaflet free margin & 34 \\
Sinus height & 19 \\
Height of commissures & 9.6 \\
\hline
\end{tabular}

